\title{
The coupled-cluster method
}

\author{
Decomposing the wavefunction of a many-particle system \\ in terms of amplitudes for exciting clusters of a finite number of particles \\ yields a versatile and high-precision tool of many-body theory.
}

\author{
Raymond F. Bishop and Hermann G. Kümmel
}

Liquids and solids, atoms and molecules, nuclei-all these clearly are interacting many-body systems. Even a nucleon may be regarded as a manyparticle system, ${ }^{1}$ not just because it is now known to consist of three quarks interacting via gluons, but because of the possibility in quantum field theory of virtual excitation of many particles from the vacuum. In the table page 56 we list some of the many-particle systems we encounter in the physical world at length scales that range from a few centimeters to a few fermis. Many of these systems exhibit phenomena-superconductivity in solids and fission in nuclei, for example-whose understanding does not follow immediately from knowledge of the constituents of the system and the interactions among the constituents, but requires new concepts and ideas. Many-body physics is the branch of theoretical physics that studies the new phenomena or "emergent properties" that arise from interactions among "elementary" constituents of a many-particle system and provides means and methods for carrying out precise calculations of such characteristic properties of these systems as may be compared with experimental results to verify hypotheses about the nature of the constituents and their interactions.

The papers by Paul A. M. Dirac and Werner Heisenberg on the symmetry properties of the wavefunction of a system of many identical particles laid the foundations of quantum many-body

Raymond F. Bishop is reader in theoretical physics at the department of mathematics of the University of Manchester Institute of Science and Technology, Manchester, England. Hermann G. Kümmel is professor of physics at the Institute for Theoretical Physics. Ruhr-Universitát Bochum, Bochum, West Germany. theory soon after the formulation of quantum mechanics. Since then, physicists working in different areas have developed a variety of methods and techniques for obtaining approximate but reliable estimates of the properties of quantum many-body systems. In the box on page 54 we briefly describe some of these techniques. We have collected in this box only those techniques that have proved to be of more general interest than the specific context or problem for which they were first developed. Indeed, we regard these techniques as constituting the "toolbox of theoretical physics" needed to attack the many-body problem, because they may be used to study any many-particle system for which the interactions among the constituents are known.

In this article we will discuss a method of quantum many-body physics, the coupled-cluster method, ${ }^{2}$ that has proved extremely useful in a wide variety of fields ranging from quantum chemistry to nuclear physics. The wavefunction of a quantum many-body system is decomposed in this method in terms of amplitudes for exciting clusters of a finite number of particles. Such a decomposition of the wavefunction is easy to interpret physically; consequently, approximations made in calculations using this method can be systematically improved. In the table on page 55 we compare the "tools" of many-body physics for range of applicability, versatility with regard to interactions and range of densities, and precision. Clearly, the CCM works for systems of both bosons and fermions quite regardless of the type and range of interaction, and yields high-precision results for the ground state as well as low-energy excited states. We regard it, therefore, as the "universal" method of theoretical many-body physics. Although there are situations-for example, very-high-density fluids with interparticle interactions containing strong and relatively long-range repulsion at small distances followed by a longer-ranged attractive tail-for which the standard form of the CCM has been found to be practically useless, we are sure that the method will soon be extended to cover these situations. We will discuss below several examples of results obtained with this method and compare them with those obtained with other methods.

\section{High-precision techniques}

The confidence we now feel in the validity of quantum mechanics is derived partly from our ability to solve the many-particle Schrödinger equation for a complicated atom or molecule to obtain, say, the energy spectrum with such precision as is needed to reproduce the experimental results accurately. Such comparisons between theoretical and experimental results are indispensable to scientific progress. To ensure that any comparison between theory and experiment in many-body physics is meaningful and free from computational uncertainties, we require that the methods of manybody theory chosen to attack a given problem be capable of yielding highprecision results. This requirement is often more important than such other considerations as the ease of implementation and the computer time needed to carry out an approximation procedure numerically.

As an example of the kind of precision needed in many-body calculations in quantum chemistry, consider the ethylene molecule. The best available estimate, obtained from the configuration-interaction method, for the total energy of this molecule is -78.35451 hartrees, whereas the simplest approximation of many-body theory-the 
a

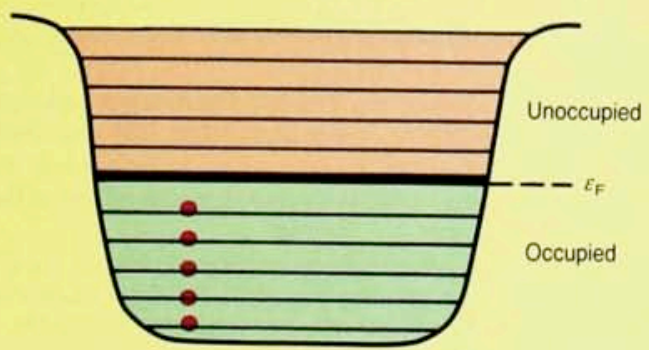

Energy levels of many-particle systems. These schematic representations depict the filled Fermi sea state $|\Phi\rangle$ (a) and a correlated pair (b), two independent correlated pairs (c) and a correlated triplet (d)

b

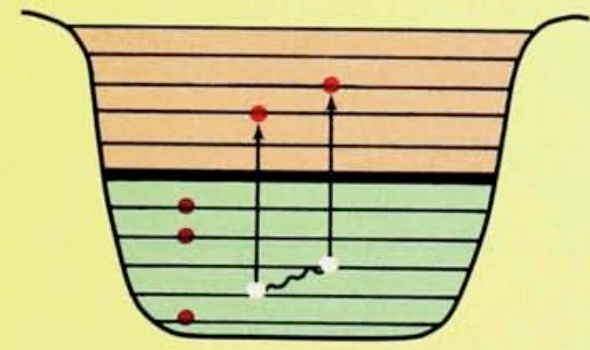
excited out of it. The many-particle wavefunction is built in the CCM by considering the probability of exciting such clusters of a finite number of particles.

Figure 1

Hartree-Fock approximation-gives -78.04520 hartrees. (A hartree, defined as $e^{2} / a_{0}$, or about $27.2 \mathrm{eV}$, is the natural unit in which to measure the energy of an electron in an atom.) Because the experimentally accessible quantity, the energy released or absorbed in a chemical reaction, is a very small fraction of molecular energies, the difference, 0.30931 hartrees, between the exact and Hartree-Fock results, called the "correlation energy," is very important. For example, the heat of formation of ethylene from hydrogen and acetylene has been measured to be only $66.99 \pm 0.4$ millihartrees (one millihartree equals 0.627 kilocalories per mole). Thus an approximate calculation of molecular energies must account for the "correlation energy" and give a result within $0.01 \%$ of the exact energy.

Besides this minimum precision demanded of a theory for a meaningful comparison of its results with experiments, there is another aspect to the need for high-precision calculations, namely, to test the predictions of a theory for any possible deviations from experimental results that might indicate new effects or phenomena not accounted for by the theory. Quantum electrodynamics, the quantum field theory of electrons and photons, has yielded the most accurate numbers of any physical theory and is a good example of the significance of highprecision calculations. The accuracy of better than one part in ten million to which experimental and theoretical values for the anomalous magnetic moment of the electron, for example, agree is irrefutable evidence in support of the theory, in spite of the divergences in it that could be overcome only with a difficult renormalization procedure.

The history of the coupled-cluster method reflects rather well some of the developments in theoretical physics in the last few decades. The method appeared in nuclear physics ${ }^{3}$ as early as 1957 , but it remained obscure for a considerable period. From our present vantage point, we see that this had much to do with the fact that the sort of high-precision calculations that the CCM allows us to do were neither fashionable nor considered necessary at that time, because our understanding of nuclear forces was then not very good. In fact, the self-consistent Brueckner theory ${ }^{4}$ is the only example from those days of a theory that considered nuclear forces beyond a simple, linear approximation and studied the effect on nuclear properties of correlations due to nonlinearities. The situation in quantum chemistry, however, has been very different: Precise determination of molecular structures and binding energies from a microscopic quantum mechanical calculation has 


\section{A many-body theorist's toolbox}

Green's function Monte Carlo method: Solves the $N$-body Schrödinger equation directly by stochastic simulation; typically used for fewer than about 250 particles.

Time-independent perturbation theory: Provides an expansion in powers of the coupling constant; leads to Goldstone diagrams. Ultimately one neglects all terms that are presumed to be unimportant or that cannot be handled.

Green's function method: A reformulation of time-dependent perturbation theory; ultimately relies on an expansion in powers of the coupling constant. Formulated as timedependent equations for matrix elements describing the propagation of groups of particles in the system; leads to Feynman diagrams.

Variational method: Computes the energy expectation value $\langle\mathbf{H}\rangle$ with a trial wavefunction typically of Jastrow form. Various techniques exist to calculate $\langle\mathbf{H}\rangle$ explicitly, for example, Monte Carlo evaluations or the "hypernetted chain" approximation.

Correlated basis function method: A systematic improvement on the variational method; enables short-range correlations to be incorporated from the start.

Configuration-interaction method: Diagonalizes the Hamiltonian in a finite subspace of the full many-body Hilbert space.

Coupled-cluster method: See main text. Iterating the basic CCM equations yields timeindependent perturbation theory-although one need not solve the CCM equations this way. been a problem of utmost importance for many quantum chemists since the development of the quantum theory of the chemical bond. These quantum chemists adopted ${ }^{5}$ the CCM from nuclear physics and developed it in many ways. When we look back at the results obtained by this method in quantum chemistry, we feel that the efforts of quantum chemists in developing the CCM have been eminently rewarded. We will discuss below several examples of condensed matter problems in which the CCM has successfully been applied. The method also has been recently adapted to attack problems in quantum field theory.

To avoid later misapprehension, we stress from the start that the CCM is not just a theory for the energies of quantum mechanical ground-states of "closed shell" systems, although this is the form in which it was invented, and which we now use for illustration purposes.

\section{The ground-state CCM}

Consider the problem of obtaining the ground-state wavefunction $|\Psi\rangle$ and energy $E$ of a system of $N$ interacting fermions described by a quantum mechanical Hamiltonian operator $\mathbf{H}$. That is, we wish to solve the time-independent Schrödinger equation

$$
\mathbf{H}|\Psi\rangle=E|\Psi\rangle
$$

for the lowest eigenvalue $E$ and corresponding eigenfunction $|\Psi\rangle$. Our purpose here is only to discuss the basic ideas of the CCM, and the exact representation we develop below for the ground-state wavefunction of a manyfermion system serves this purpose well. These ideas have been extended ${ }^{2}$ to obtain low-lying excited states, the dynamical response to external perturbations and the exact sum rules the response functions obey.

If the fermions in a many-body system do not interact with one another, then the only constraint on the manyparticle wavefunction is the Pauli exclusion principle, which requires each fermion to be in a different singleparticle eigenstate. The $N$-body ground state in this simple case is obtained by filling the $N$ lowest singleparticle states as indicated in figure 1a. The wavefunction is a sum of products of the one-body wavefunctions of the "occupied states." The $N$ ! terms in this sum differ only by pairwise permutations of the single-particle coordinates and their signs are chosen so as to maintain overall antisymmetry, demanded by Fermi-Dirac statistics, with respect to interchange of any two particles. This sum is most compactly expressed in a determinantal form and is called the "Slater determinant." The set of occupied levels is often referred to as the "filled Fermi sea." In the Hartree-Fock approximation that we mentioned earlier, the interacting many-particle system is replaced ${ }^{4}$ by an "effective" noninteracting one, in which each particle moves independently in a potential well that is determined self-consistently by the average motion of all the particles. Determination of the self-consistent potential and solution of the Schrödinger equation for single-particle eigenstates in it are the major steps in working out this approximation; once the single-particle states in the selfconsistent potential are known, the ground-state wavefunction and energy are simply obtained by the method outlined above for a system of noninter- acting particles.

We now consider how the correlations induced by interactions modify this picture of the ground state as a filled Fermi sea. The first thing one may imagine happening is that two particles interact and lift themselves out of the Fermi sea, so that after the interaction both particles are in orbitals that in the previous simplified picture were unoccupied (see figure $1 \mathrm{~b}$ ). This process may be described by a quantum mechanical operator $\mathbf{S}_{2}$, which acts on the Fermi sea wavefunction $|\Phi\rangle$ to produce the wavefunction $\mathbf{S}_{2}|\Phi\rangle$, which describes two particles outside the Fermi sea (and consequently two "holes" inside it) and all remaining $N-2$ particles in their previous orbitals. But it may also happen that two pairs of particles do this completely independently, as illustrated in figure 1c. Clearly, this process is described by applying the operator $\mathbf{S}_{2}$ twice, but with the proviso that we must include a statistical weighting factor (or multiplicity) of $1 / 2$ to avoid counting the pairs twice. The resulting contribution to the wavefunction is $1 / 2 \mathbf{S}_{2}^{2}|\Phi\rangle$. This process of independent excitation of pairs out of the Fermi sea may be continued to obtain a contribution $(1 / m !) \mathbf{S}_{2}{ }^{m}|\Phi\rangle$ for the amplitude describing the excitation of $m$ independent pairs. By the principle of linear superposition, the total amplitude for the excitation of an arbitrary number (including zero) of independent pairs is

$$
\sum_{m=0}^{\infty} \frac{1}{m !} \mathbf{S}_{2}^{m}|\Phi\rangle=\mathrm{e}^{\mathbf{S}}|\Phi\rangle
$$

Next we consider processes involving simultaneous excitation of three particles, as in figure $1 \mathrm{~d}$. These may be 


\begin{tabular}{|c|c|c|c|c|c|c|c|}
\hline $\begin{array}{l}\text { Main applications } \\
\text { Atoms } \\
\text { Molecules } \\
\text { Solids } \\
\text { Gases } \\
\text { Fluids } \\
\text { Nuclei } \\
\text { Elementary particles }\end{array}$ & $\begin{array}{c}\text { GFMC } \\
(\bullet) \\
(\bullet) \\
\text { (Mostly bosons) } \\
\text { (Mostly bosons) }\end{array}$ & $\begin{array}{l}\text { TIPT } \\
\bullet \\
\bullet \\
\bullet\end{array}$ & $\begin{array}{l}\bullet \\
\bullet \\
(\bullet) \\
\bullet\end{array}$ & $\begin{array}{l}\text { VAR } \\
\bullet \\
\bullet \\
\bullet\end{array}$ & : & $\begin{array}{l}\text { Cl } \\
\bullet \\
\bullet\end{array}$ & $\begin{array}{c}\text { CCM } \\
\bullet \\
\bullet \\
\bullet \\
\bullet \\
\bullet \\
\bullet\end{array}$ \\
\hline $\begin{array}{l}\text { Versatility } \\
\text { Type of interaction } \\
\text { handled }\end{array}$ & $\begin{array}{l}\text { Simple (minimal } \\
\text { state dependence) }\end{array}$ & $\begin{array}{l}\text { Weak only (or weak } \\
\text { medium-range } \\
\text { interaction outside } \\
\text { a strong core) }\end{array}$ & All & $\begin{array}{l}\text { Simple (minimal } \\
\text { state dependence) }\end{array}$ & All & Soft only & All \\
\hline $\begin{array}{l}\text { Range of densities } \\
\text { (for a given } \\
\text { interaction) }\end{array}$ & High & $\begin{array}{l}\text { Low (medium for } \\
\text { systems with } \\
\text { short- to medium- } \\
\text { range interactions) }\end{array}$ & Medium & High & High & Medium & $\begin{array}{l}\text { Medium (high in } \\
\text { extended version) }\end{array}$ \\
\hline $\begin{array}{l}\text { Precision for given } \\
\text { computer time and } \\
\text { for a moderately } \\
\text { difficult interaction }\end{array}$ & High & $\begin{array}{l}\text { Low (medium for } \\
\text { partially re-summed } \\
\text { series) }\end{array}$ & $\begin{array}{l}\text { Medium (high } \\
\text { for QED) }\end{array}$ & Medium & Medium & Medium & High \\
\hline Intrinsically exact & $\bullet$ & $\bullet$ & $\bullet$ & & $\bullet$ & $\bullet$ & $\bullet$ \\
\hline Quantities obtained & Ground state & Ground state & S-matrix, sum rules & Ground state & $\begin{array}{l}\text { Ground state, } \\
\text { low excited states }\end{array}$ & $\begin{array}{l}\text { Ground state, } \\
\text { low excited states }\end{array}$ & $\begin{array}{l}\text { Ground state, } \\
\text { low excited states, } \\
\text { sum rules }\end{array}$ \\
\hline $\begin{array}{l}\text { GFMC: Green's function } \\
\text { TIPT: Time-independent } \\
\text { GF: Green's function met } \\
\text { VAR: Variational method }\end{array}$ & $\begin{array}{l}\text { Monte Carlo method } \\
\text { perturbation theory } \\
\text { thod }\end{array}$ & $\begin{array}{l}\text { CBF: Correlated ba } \\
\text { CI: Configuration-ir } \\
\text { CCM: Coupled-clus }\end{array}$ & $\begin{array}{l}\text { asis function method } \\
\text { interaction method } \\
\text { ster method }\end{array}$ & & & & \\
\hline
\end{tabular}

described by a contribution $\mathbf{S}_{3}|\Phi\rangle$ to the exact wavefunction. As in the case of simultaneous excitation of several pairs of particles, there will be a contribution $(1 / n !) \mathbf{S}_{3}{ }^{n}|\Phi\rangle$ from the simultaneous excitation of $n$ independent triplets. Now we must also account for the possibility of simultaneous excitation of pairs and triplets: Again by linear superposition, the amplitude for independent simultaneous excitation of $m$ pairs and $n$ triplets from the Fermi sea is $(1 / m ! n !)$ $\times \mathbf{S}_{2}{ }^{m} \mathbf{S}_{3}{ }^{n}|\Phi\rangle$. Here we do not need to worry about the ordering of the product of operators $\mathbf{S}_{2}$ and $\mathbf{S}_{3}$ because they describe independent processes and therefore commute. Summing over all possible values of $m$ and $n$ then leads to the amplitude

$$
\mathrm{e}^{\left(\mathbf{S}_{2}+\mathbf{S}_{3}\right)}|\Phi\rangle
$$

for the total effect of all pair and triplet excitations. Proceeding in this way with the excitation of clusters of 4 , $5, \ldots, N$ particles we arrive at a wavefunction

$$
\mathrm{e}^{\left(\mathbf{S}_{2}+\mathbf{S}_{3}+\ldots+\mathbf{S}_{N}\right)}|\Phi\rangle
$$

In our discussion of the wavefunction, we did not consider the possibility that in the interaction of any subset of particles only one of them is finally lifted out of the Fermi sea, whereas the others remain inside. This is because of a theorem ${ }^{4}$ proved by David Thouless that states that the most general determinantal wavefunction $\left|\Phi^{\prime}\right\rangle$ not orthogonal to a given Slater determinant Ф) has the form

$$
\left|\Phi^{\prime}\right\rangle=\exp \left(\mathbf{S}_{1}\right)|\Phi\rangle
$$

for some suitable choice of the operator $\mathbf{S}_{1}$, which acts on $|\Phi\rangle$ to produce a "particle-hole" excitation. In other words, the effect of allowing single particles to be independently elevated above the Fermi sea is equivalent to changing the single-particle orbitals that make up the Slater determinant.

In practical applications of the CCM it is often desirable that the starting determinantal wavefunction be as close as possible to the exact wavefunction, so that the correlations induced by operators $\mathbf{S}_{2}, \mathbf{S}_{3}, \ldots, \mathbf{S}_{N}$ are small and may be treated perturbatively. Therefore, the single-particle orbitals are often chosen in such a way that the overlap of the Slater determinant with the exact wavefunction is maximized. If this condition is satisfied, it is no longer necessary to consider singleparticle excitations separately.

The representation

$$
\begin{gathered}
|\Psi\rangle=\mathrm{e}^{\mathbf{S}}|\Phi\rangle \\
\mathbf{S}=\sum_{n=1}^{N} \mathbf{S}_{n}
\end{gathered}
$$

we have obtained for the $N$-fermion wavefunction is quite general since we made no approximation in considering the effect of interactions. But there is one caveat. The wavefunction of equation 2 describes the ground state of the interacting many-body system only if the overlap of the starting Slater determinant with the exact wavefunction is nonzero; if the overlap is zero in spite of the best possible choice of the singleparticle orbitals, it may mean that the ground state of the interacting system has different symmetries from those of the noninteracting one and cannot be obtained from the latter. However, there are extensions of the CCM to handle such cases. ${ }^{2}$

John Hubbard ${ }^{6}$ may have been the first to notice that the operator generating the wavefunction of a quantum many-body system has an exponential form, like the one we obtained in equation 2. Hubbard's ideas also apply to the representation of eigenstates in quantum field theory. ${ }^{7}$ This gives us a reason to hope that the ideas of the CCM that we used to obtain equation 2 might also prove useful in quantum field theory.

This exponential representation may be regarded as an expansion of the exact wavefunction in a complete orthonormal basis, but it is more useful to keep in mind the arguments we used to 


\section{Examples of many-body systems}

\begin{tabular}{|c|c|c|c|}
\hline System & Particles & Interaction & Infinite Limit \\
\hline Molecular clusters & $\mathrm{CO}_{2}$ molecules, for example & $\begin{array}{l}\text { Van der Waals type forces (repulsive core } \\
\text { plus attractive tail) }\end{array}$ & Gases, fluids, molecular crystals \\
\hline \multirow{7}{*}{$\begin{array}{l}\text { Rare gas atom clusters } \\
\text { Metallic atom clusters } \\
\text { Atoms and molecules } \\
\text { Nuclei }\end{array}$} & He or Ar atoms, for example & Interatomic potentials such as van der Waals & Rare gas liquids, crystals \\
\hline & $\mathrm{Na}$ or $\mathrm{Ag}$ atoms, for example & Interatomic potentials & Metallic crystals \\
\hline & Electrons and nuclei & Coulomb potentials & Infinite Thomas-Fermi atom \\
\hline & Alpha particles $(A=2 Z)$ & $\begin{array}{l}\text { Two-body phenomenological potentials (repulsive core } \\
\text { plus attractive tail); also sometimes } 3 \text {-body potentials }\end{array}$ & Alpha matter \\
\hline & Nucleons & $\begin{array}{l}\text { Two-body phenomenological potentials (repulsive core } \\
\text { plus attractive tail); also sometimes } 3 \text {-body potentials }\end{array}$ & "Standard" nuclear matter \\
\hline & Nucleons and mesons & Meson exchange & Nuclear matter as "baryon-meson soup" \\
\hline & Quarks and gluons & Hints from QCD & Quark matter \\
\hline Nucleons and other had & Quarks and gluons & QCD & Already there \\
\hline
\end{tabular}

obtain it and regard it as a cluster decomposition of quantum mechanical amplitudes. Such an interpretation is very useful in practical applications of the CCM (see below). Moreover, when this cluster picture is combined with the probabilistic interpretation of quantum mechanics, the exponential form of the operator acting on the Slater determinant, and the representation of the operator $\mathbf{S}$ in the exponent as a linear sum of operators generating excitations involving clusters of finite numbers of particles, becomes clear: This form of the many-particle wavefunction is a direct consequence of the law of probability theory that states that the probability of statistically independent processes is a product of the probabilities of the individual processes. The partition function of classical statistical mechanics, from which all thermodynamic functions may be derived, is also computed in the UrsellMayer cluster expansion ${ }^{8}$ by studying independent clusters of a given size, and it also has an exponential form provided the statistical multiplicities of various cluster processes are properly taken into account.

The problem of finding the groundstate energy and wavefunction of the $N$-fermion system is reduced in the CCM to computing the operators $\mathbf{S}_{n}-$ or, in practice, their matrix elements. The configuration-interaction method, by contrast, uses combinations of clusters instead of the clusters described by the $\mathbf{S}_{n}$ themselves, with the result that numerical instabilities arise for large particle numbers $N$. In the perturbation methods, the quantity of interest is expanded in powers of an effective "coupling constant" of the interactions in the system, which works best when the coupling constant is small. Only a few realistic systems of interest, such as quantum electrodynamics, satisfy this condition, however. In other perturbative calculations one must have some intuition about the important terms in the perturbation series. There exist many examples where very reasonable-sounding arguments for what "the next most important set of terms or diagrams" is have simply proved either incorrect or-what is often tantamount to the same thingincomplete. We will discuss below how to generate approximate solutions to the CCM equations and compare these approximations with those in perturbation theory.

The CCM equations for the matrix elements of $\mathbf{S}_{n}$ are easily found by projecting the Schrödinger equation

$$
\mathrm{e}^{-\mathrm{s}} \mathrm{H} \mathrm{e}^{\mathrm{s}}|\Phi\rangle=E|\Phi\rangle
$$

onto the complete $N$-body space spanned by the Fermi sea states and those states obtained by creating $n$ general particle-hole excitations out of it. This yields a series of coupled equations, each of which contains a finite number of terms. The first equation in this series immediately yields an expression for $E$. Due to the special form of the Schrödinger equation given in equation (3), the remaining equations do not involve the energy $E$ or other macroscopic terms, and represent a truly microscopic decomposition of the Schrödinger equation into a set of coupled equations that describe the dynamics of the $n$-body clusters. These equations are intrinsically nonlinear.
In the familiar example of pairwise interactions, matrix elements of the operators $\mathbf{S}_{\text {, }}$ may appear raised up to the fourth power, and the equation for the matrix elements of $\mathbf{S}_{n}$ is coupled to equations for all $\mathbf{S}_{i}$ with $i$ less than or equal to $n+2$. By formally iterating these equations in powers of the interaction potential, one may immediately regain the individual terms of the timeindependent perturbation theory, expressed in terms of the linked-cluster expansion of Jeffrey Goldstone. ${ }^{9}$ We would like to stress, however, that this is never necessary for the actual solution of our equations. In fact, the perturbative expansion very often shows divergences, even when the CCM equations have solutions. This happens because the CCM achieves a natural grouping of terms in the perturbation series that overcomes the divergences in individual terms.

\section{Approximation schemes}

Few realistic models of many-particle systems are exactly solvable and, in spite of the exact formulation we discussed above for the ground-state energy and wavefunction in the CCM, we have to resort to some approximation schemes to solve the coupled equations. We noted above how the coupled equations of the CCM generate the terms of the time-independent perturbation theory - a very widely used approximation scheme in many-body theory that works best when the interactions are weak. However, a great advantage of the CCM is that the physical interpretation of the wavefunction readily lends itself to approximation schemes that are motivated by the physics of the 


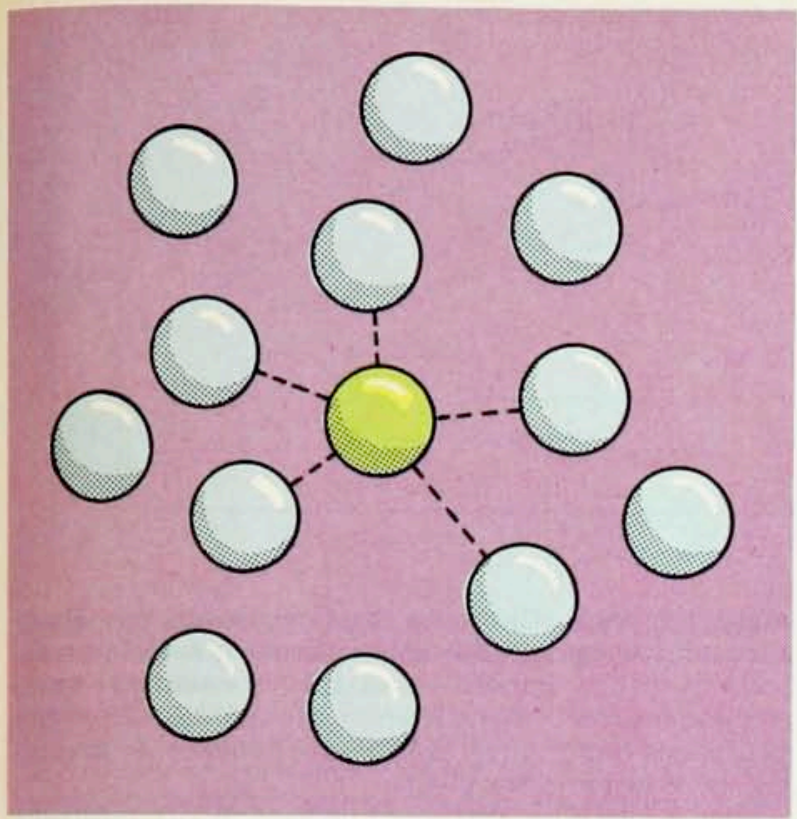

Interactions. In a many-body system a given particle typically interacts, on average, with relatively few other particles, unless the entire system is strongly phase ordered in some way. Here a cluster of five immediate neighbors surrounds the green particle. In many real systems such a "correlation cluster" effectively screens out the central particle's interactions with the other particles.

Figure 2

problem rather than by mathematical convenience. For instance, one may argue that for relatively "low density" or relatively "weakly interacting" systems, only comparatively rarely do more than a few particles come together to lift themselves simultaneously out of the Fermi sea-the actual number may be as low as 2 or 3 (see figure 2). This results in the so-called "natural," or $\mathrm{SUB} n$, approximation hierarchy, in which all clusters with more than $n$ particles are neglected and the remaining $n$ coupled equations are solved as accurately as possible. This approach has proven extremely successful for atomic and molecular systems. The SUB2 approximation-the next step beyond the Hartree-Fock approximation if the single-particle excitations are absorbed into the Hartree-Fock orbitals-contains as drastic sub-approximations to itself such other familiar approximations as

- The Bethe-Goldstone approximation that sums the so-called particleparticle ladder diagrams that describe the repeated scattering of two particles inside the many-body medium

- The analogous Galitskii approximation that also includes all hole-hole and mixed particle-particle and hole-hole ladder terms

- The random-phase approximation. We note that the SUB2 approximation also contains terms that include in the last two cases, for example, the effects in the intermediate scattering states. These terms arise from the fact that the particles and holes both move in the (self-consistent) potentials due to the rest of the medium. Thus, the whole of the usual Brueckner-Bethe-Goldstone theory applied so often to nuclear matter and finite nuclei is also imbedded in the SUB2 approximation. Furthermore, the SUB2 approximation is richer even than the fully self-consistent union of all the above approximations: It also contains classes of particle-hole ladder terms and extra exchange terms to preserve the overall antisymmetry imposed by the fermion statistics-but for fuller details we must refer the reader to the literature. ${ }^{10}$

There are a variety of other approximation schemes, some tailor-made to deal with a specific situation: For example there is the so-called hard-core scheme $(\mathrm{HC}-\mathrm{SUB} n)$ to deal with interparticle forces that contain a very strong repulsive core, as in nuclear physics; another scheme has been applied with great success to systems, such as electrons in solids, in which the interparticle forces are long range. Instead of describing these and other approximations in detail, let us just say that there now exists a considerable body of experience that one may refer to when selecting the approximation within the CCM that will be most suitable for a given problem.

\section{Results from CCM}

We now review some of the results obtained by using the CCM, and examine how it compares with other methods in solving real problems.

Quantum chemistry. The coupled-cluster method is now regarded as one of the standard methods of quantum chemistry, and it is the method of first choice in nearly all cases where it can be employed. We quote here only one example of the many applications of the CCM in quantum chemistry: the problem of obtaining the binding energy of the $\mathrm{LiH}$ molecule. But this example illustrates quite well that one can overcome most of the difficulties that one encounters in CCM calculations.

For a molecule with a complex geometry, even the determination of a good starting determinantal wavefunction Ф) via, for example, the Hartree-Fock self-consistent field equations is already a major task. Solving this problem by itself constitutes a "major industry" in quantum chemistry, but these efforts cannot yield high-precision numbers because they do not include the correlations induced by $\mathbf{S}_{2}$, $\mathbf{S}_{3}, \ldots$ In many cases the correlation energy is small and can be adequately handled by relatively straightforward perturbation theory, if a good starting determinant is available. The full power of the CCM is not needed in such cases. But only the CCM seems to work efficiently when the correlations are large. Lithium hydride is an example of a many-body system for which it is very difficult to obtain a good starting determinant or in which the matrix elements of some of the $\mathbf{S}_{n}$ are intrinsically large. We call such systems "decisive systems." We hope that the good results obtained for $\mathrm{LiH}$ will convince the reader of the true power of the CCM.

The short-distance singularity of the Coulomb interaction between electrons, which has the effect of producing cusps in the wavefunction, is also a feature that needs special attention when choosing the single-particle orbi- 
tals for the basis in which the equations of the CCM are expanded. In quantum chemistry, the standard choice for the basis states is the complete set of orbitals from the lowest of which the Slater determinant itself is composed. However, the singular cusps in the wavefunction are not suitably represented by an expansion in a small number of single-particle orbitals and often cause instabilities in numerical solutions of the equations of the CCM. To circumvent this problem, the newer techniques employ the so-called geminal expansions, in which the singleparticle orbitals are replaced by explicitly correlated functions. The typical geminal thus comprises the product of a pair of single-electron orbitals together with a factor (typically of Gaussian form) that depends explicitly on the distance between the two electrons. This change in basis has the effect of reducing by many orders of magnitude the number of terms needed for a given level of accuracy in representing the cluster amplitudes. Consequently the amount of computer time needed for a given accuracy is also reduced, although not by the same factor because the geminal basis is itself more complicated. Results for the $\mathrm{LiH}$ molecule that we discuss below were obtained using a geminal basis. ${ }^{11}$

At the SUB2 level of approximation the CCM yields 8.06867 hartrees for the total binding energy of the $\mathrm{LiH}$ molecule, compared with the exact value of $8.07036 \pm 0.0001$ hartrees. (The exact value is obtained from the best experimental and theoretical estimates.) The Hartree-Fock energy accounts for 7.98716 hartrees, so that out of a correlation energy of -83.2 millihartrees, the CCM misses only 1.69 millihartrees. Thus the CCM succeeds in achieving accuracy on the order of a millihartree already at the SUB2 level. As discussed in the beginning, such an accuracy is the goal of many-body calculations in quantum chemistry. Furthermore, we know how to improve the calculation systematically, by considering the correlations induced by higher $\mathbf{S}_{n}$

As an example of how the computer time needed in CCM calculations compares with other methods, we return to the ethylene molecule. This is also a decisive system in the sense defined earlier. Increasing the distance between the two carbon atoms by approximately $50 \%$ beyond the equilibrium value causes the atomic configuration to change from a planar to a "twisted" nonplanar structure. In other words the system even in equilibrium is nearly degenerate, and there are two quite different Slater determinants in competition with each other. For this molecule there exists a benchmark configuration-interaction calculation using more than a million configurations, which naturally consumes a considerable amount of computer time. We quoted above the extremely good results obtained from this calculation. The CCM yields practically the same accuracy with roughly a tenth of the computer time. ${ }^{12}$ This example is now quite typical in quantum chemistry applications. Of course, had the same computer time been allotted to the CCM calculation, the increase in accuracy over the configuration-interaction calculation would have been appreciable. Conversely, even if the time were reduced by a factor of 10 , the CCM calculations would still yield results of good quality.

Nuclear physics. To demonstrate the efficiency of the CCM in solving problems in nuclear physics, we consider a problem for which exact results also exist: a model for the alpha particle that uses for the internucleon force a purely central (and hence grossly oversimplified) form known as the MalflietTjon $\mathrm{V}$ force. The four nucleons of the alpha particle move in this state-independent force as if they were bosons, because they must be in different spinisospin states. In this special case the four-body Schrödinger equation could be solved by Green's function Monte Carlo techniques to provide a benchmark result that is essentially exact, apart from small statistical errors that may in principle be made as small as we please. ${ }^{13}$

In the box on page 60 we compare the "exact" Green's function Monte Carlo results with the CCM results, HC-SUB2 and HC-SUB3, obtained at the second and third levels, respectively, of the approximation scheme already mentioned, and also with the results of a typical good variational calculation. We note that the variational calculation, although it is quite accurate and efficient to carry out on a computer, cannot be regarded as a high-precision method, because to improve on the results for this method quoted in the box, by using correlated basis function techniques, for example, dramatically extends both its difficulty and the time needed for its implementation. We may also point out here that there are formal techniques available to solve the quantum-mechanical four-body problem exactly. These techniques have been applied to this model, giving an energy of only $-28 \mathrm{MeV}$, rather than the benchmark result of -31 $\mathrm{MeV}$. This "exact" calculation took many hours of computing time (on the scale used in the table on page 60 and used some of the best numerical approximations that could be implemented in the available time. There is a wider lesson for many-body theory here: Successive levels of approximation of a high-precision method that is capable of systematic improvement may in practice be preferable to a oneshot implementation of an exact method.

Electron gas. The electron gas, or "jellium," is an idealized infinite homogeneous system of electrons together with an inert background of homogeneous positive charge to ensure charge neutrality, and provides a simple model for conduction electrons in solids. It may well lay claim to being the most 


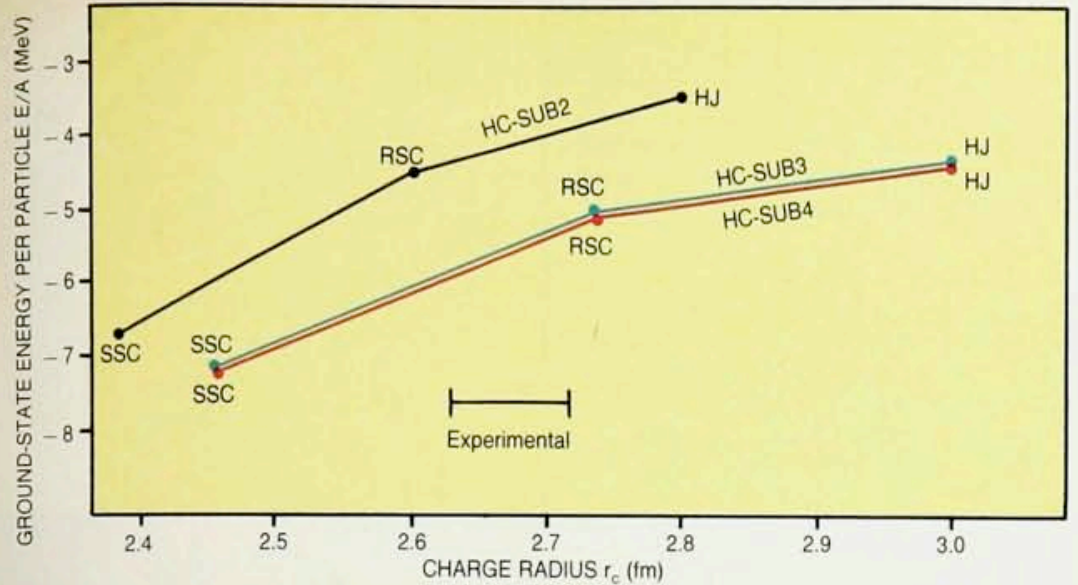

The ground-state energy per particle, $E / A$, for the closed-shell nucleus $O^{16}$ as a function of the charge radius $r_{c}$. The theoretical CCM results were obtained by including correlated cluster amplitudes $\mathbf{S}_{n}$ up to order $n=2$ (black), 3 (blue) and 4 (red). The two-body internucleon potential is not definitively known, so each calculation at a given order was performed for three phenomenological potentials that accurately reproduce the experimental two-body data: a super-soft-core potential (SSC), the Reid soft-core potential (RSC) and the Hamada-Johnston hard-core potential $(\mathrm{HJ})$. The sequence of approximations converges very rapidly. That none of the two-body potentials reproduces the experimental value suggests that nuclei are not many-nucleon systems in which the nucleons interact only via two-body potentials.

Figure 3

studied of all quantum many-body problems. A Green's function Monte Carlo calculation with small statistical errors (and, to the best of our belief, negligible systematic errors) is even available for this system, made possible by the special nature of the Coulomb interaction. (Few many-fermion systems have been studied using the Monte Carlo methods.)

As already mentioned, a rather sophisticated approximation scheme has been found in the CCM to deal with the long range of the Coulomb potential. This approximation also makes it possible to include the important three- and four-body cluster effects without actually solving the CCM equations for three- and four-body subsystems. Because of strong correlations in this system, three- and four-body cluster effects are very imporant in highprecision applications of the CCM. In the box on page 60 we compare results for the correlation energy at various values of the density (parameterized by the dimensionless coupling constant $r_{\mathrm{s}}$, the average interelectron spacing in units of the Bohr radius). We have not quoted in this table results for the electron gas obtained by methods other than the Green's function Monte Carlo. The numerical CCM results are in fact in better agreement with the "exact" Green's function Monte Carlo data than those of any other numerical calculation.

Anharmonic oscillators. Various onedimensional anharmonic oscillators have been widely studied ${ }^{14}$ lately because they provide simple models of nonlinear quantum field theories. The Hamiltonian for the well-studied example of the quartic anharmonic oscillator is

$$
\mathbf{H}=1 / 2\left(\mathbf{p}^{2}+\mathbf{q}^{2}\right)+\lambda \mathbf{q}^{4}
$$

where $\mathbf{q}$ is the coordinate operator on the infinite line and $\mathbf{p}$ is the corresponding canonical momentum. Carl Bender and Tai T. Wu have shown that perturbation theory diverges in this case for all values of the coupling constant $\lambda$. The problem can be solved by direct diagonalization, but good accuracy requires very large matrices and corresponding computer power. In the box on page 60 we indicate the results obtained with the CCM in various approximations including only $\mathbf{S}_{2}$ and $\mathbf{S}_{4}$-clusters of odd numbers of particles cannot occur in this case. The CCM equations are so simple that the results quoted in the box, except perhaps for the last one, can be obtained on a good programmable pocket calculator.

\section{CCM and the nuclear force}

As an example of the kind of insights that precise and detailed calculations made possible by the CCM provide, we return to nuclear physics and examine the status of our understanding of nuclear forces in nuclei. It has been recognized for a long time that meson exchanges between nucleons (for the sake of simplicity, we ignore quarks here) play an important role in holding the nucleus together. However, because these processes are not well understood, it has been common prac- tice since the early days of nuclear phenomenology to use two-body potentials to represent the interaction mediated by meson exchange. These twobody potentials were obtained from experiments on nucleon-nucleon scattering and although they reproduce the two-body scattering data quite accurately, they ignore the possibility of new exchange effects-in the threenucleon problem, for example-that cannot be reduced to a sum of two-body exchanges.

Is the nuclear force inside nuclei well represented by these two-body potentials? Or are there many-body effects that even the best of two-body potentials cannot account for? These questions could not be definitively answered until about ten years ago because calculations of nuclear properties using two-body potentials were not accurate enough to determine whether any discrepancy between calculated and measured values was due to the approximations made in the calculations or was evidence for many-body effects. But there now exist exact solutions of the three-body problem ${ }^{15}$ as well as highprecision CCM calculations on various heavier nuclei ${ }^{2}$ that fail to reproduce experimental data even when the best available two-body potentials are used. In figure 3 we show the results obtained from one such calculation for the ground-state energy of the closed-shell nucleus $\mathrm{O}^{16}$. The CCM shows good convergence in this case-truncating the method at three- and four-particle clusters, respectively, gives almost identical results. The large difference between experimental and calculated values in figure 3 leads to the inescapable conclusion that nuclei are not many-nucleon systems in which the nucleons interact via two-body potentials. This may have been suspected for a long time but these calculations offer the first definitive and quantitative evidence that a picture of the nucleus based on two-body forces between nucleons will not serve as an adequate 


\section{Results from the coupled-cluster method}

We present here comparisons of the results for three problems: the energy of an alpha particle, the correlation energy of an electron gas and the energy levels of an anharmonic oscillator. In each case the coupled-cluster method gives results that are very good indeed because an immense amount of (possibly collective) virtual excitations have been accounted for.

\section{Nuclear physics}

The table below shows the energy $E$ and mass radius $r_{m}$ for the alpha particle calculated with the so-called Malfliet-Tjon V potential, using various methods. The calculations are described in the text, and the column labeled "time" gives an indication of the computing time needed to implement them on a mainframe (CDC7600) computer.

\begin{tabular}{lccr} 
Method & \multicolumn{1}{c}{$\begin{array}{c}\text { Energy } \\
(\mathrm{MeV})\end{array}$} & $\begin{array}{c}\text { Mass radius } \\
(\mathrm{fm})\end{array}$ & $\begin{array}{r}\text { Time } \\
(\mathrm{sec})\end{array}$ \\
Green's function Monte Carlo & $-31.3 \pm 0.2$ & $1.36 \pm 0.01$ & 3600 \\
Coupled-cluster (HC-SUB2) & -30.24 & 1.41 & 20 \\
Coupled-cluster (HC-SUB3) & -31.24 & 1.36 & 300 \\
Variational & -30.7 & - & 30
\end{tabular}

\section{Electron gas}

We compare the correlation energy of the three-dimensional electron gas computed with the coupled-cluster method with that computed with the Green's function Monte Carlo method, for various values of the dimensionless coupling constant $r_{\mathrm{s}}$ defined in the text. The energies are given in millirydbergs.

\section{Method}

Green's function Monte Carlo

$$
\begin{array}{cccccc} 
& \multicolumn{4}{c}{\text { Coupling constant } r_{\mathbf{s}}} \\
\mathbf{1} & \mathbf{2} & \mathbf{5} & \mathbf{1 0} & \mathbf{2 0} \\
-120 & -90.2 & -56.3 & -37.22 & -23.00 \\
-122 & -90.4 & -56.0 & -37.0 & -23.6
\end{array}
$$

Coupled-cluster (SUB4)

\section{Anharmonic oscillator}

We compare the maximal deviations from the exact results in the energies of the ground and first two excited states of the quartic anharmonic oscillator with the coupling constant $\lambda$ positive and less than 1000 , for various coupled-cluster calculations.

- The standard coupled-cluster method SUB4 built on the vacuum state $|0\rangle$, that is, $|\Psi\rangle=\mathrm{e}^{\mathrm{s}}|0\rangle$, gives an error less than $3 \%$.

- Starting with a trial state $|\Phi\rangle=\mathrm{e}^{\mathrm{s}}=|0\rangle$ and optimizing for minimal energy, then using SUB4 on $|\Phi\rangle$, that is, $|\Psi\rangle=\mathrm{e}^{\mathrm{s}_{2}+\mathrm{s}} \cdot|\Phi\rangle$, gives an error less than $0.25 \%$.

- Starting with a trial state $|\Phi\rangle=\mathrm{e}^{\mathrm{s}}=|0\rangle$ and optimizing for minimal energy, followed by diagonalization of a $3 \times 3$ matrix in the obvious basis built on $|\Phi\rangle$, gives an error less than $1 \%$.

- Starting with a trial state $|\Phi\rangle=\mathrm{e}^{s_{z}}|0\rangle$ and optimizing for minimal energy, followed by diagonalization of a $10 \times 10$ matrix in the obvious basis built on $|\Phi\rangle$, gives an error less than $10^{-4} \%$.

model even for the ground-state properties of the simplest nuclei.

We hope that the few results we discussed above have convinced the reader of the power and versatility of the CCM. The method also has a great aesthetic appeal: At a time when the increasing diversity of phenomena that fall within the scope of physics has forced physicists to specialize in ever smaller subfields, it is heartening to have a method whose ideas have proved fruitful in almost all studies of manybody systems. We would like to conclude by saying that the method may also be extended into the arena of elementary particle physics and field theory. Of course, as has been the case in nuclear physics, it may take some time before the method is fully established and accepted in this field. As in other areas of physics in the past, there is certainly a need now in elementary particle physics for results accurate at the $1 \%$ level. We are sure that when suitably developed, the CCM will also succeed here as a powerful tool.

\section{References}

1. For example, see G. E. Brown, M. Rho, PHYSICS TODAY, February 1983, p. 24.

2. For reviews of the CCM see H. Kümmel, K. H. Lührmann, J. G. Zabolitzky, Phys. Rep. 36C, 1 (1978); V. Kvasnička, V. Laurinc, S. Biskupič, Phys. Rep. 90, 160 (1982); H. Kümmel, in Nucleon-Nucleon Interaction and Nuclear Many. Body Problems, S. S. Wu, T. T. S. Kuo, eds., World Scientific, Singapore (1984), p. 46 ; R. F. Bishop, in Nucleon-Nucleon Interaction and Nuclear Many-Body Problems, S. S. Wu, T. T. S. Kuo, eds., World Scientific, Singapore (1984), p. 604; J. Arponen, Ann. Phys. (N. Y.) 151 , 311 (1983).

3. F. Coester, Nucl. Phys. 7, 421 (1958), F. Coester, H. Kümmel, Nucl. Phys. 17, 477 (1960).

4. See, for example, A. L. Fetter, J. D. Walecka, Quantum Theory of Many-Particle Systems, McGraw-Hill, New York (1971); D. J. Thouless, The Quantum Mechanics of Many-Body Systems, Academic, New York (1961).

5. J. Čižek, J. Chem. Phys. 45, 4256 (1966);
Adv. Chem. Phys. 14, 35 (1969).

6. J. Hubbard, Proc. R. Soc. London, Ser. A 240, 539 (1957).

7. See, for example, S. S. Schweber, An Introduction to Relativistic Quantum Field Theory, Harper and Row, New York (1964).

8. J. E. Mayer, M. G. Mayer, Statistical Mechanics, 2nd ed., Wiley, New York (1977).

9. J. Goldstone, Proc. R. Soc. London, Ser. A 239, 267 (1957).

10. R. F. Bishop, K. H. Lührmann, Phys. Rev. B 17, 3757 (1978); Phys. Rev. B 26, 5523 (1982). K. Emrich, J. G. Zabolitzky, Phys. Rev. B 30, 2049 (1984).

11. K. Szalewicz, J. G. Zabolitzky, B. Jeziorski, H. J. Monkhorst, J. Chem. Phys. 81, 2723 (1984).

12. W. D. Laidig, G. D. Purvis, R. J. Bartlett, J. Phys. Chem. 89, 2161 (1985).

13. J. G. Zabolitzky, M. H. Kalos, Nucl. Phys. A 356, 114 (1981).

14. C. S. Hsue, J. L. Chern, Phys. Rev. D 29, 643 (1984).

15. W. D. Glöckle, The Quantum Mechanical Few-Body Problem, Springer-Verlag, Heidelberg (1983). 